\title{
Current status of emergency department attending physician ultrasound credentialing and quality assurance in the United States
}

\author{
Devjani Das ${ }^{1 *}$, Monica Kapoor ${ }^{1}$, Cara Brown ${ }^{1}$, Afoma Ndubuisi ${ }^{1}$ and Sanjey Gupta ${ }^{2}$
}

\begin{abstract}
Background: The use of emergency ultrasonography (EUS) has gained much popularity in the past few decades, and is now a mainstay of diagnostic decision-making. This expanded use is now highlighting the substantial issue of individual hospitals in credentialing its emergency medicine attending physicians in EUS in the United States. This issue is also of importance as more hospitals are now requesting reimbursements for emergency ultrasounds. The objective of this study is to gain an understanding of how many emergency departments are currently credentialing its attending staff in EUS, what the internal structure and staffing are of these emergency departments, and how they are currently performing quality assurance of the ultrasounds performed.
\end{abstract}

Methods: This was a cross-sectional, web-based survey sent to 160 ACGME-accredited EM residency programs from July 2013 to November 2013. The survey consisted of 23 questions regarding: (1) number of emergency medicine attendings on staff, (2) presence of an EUS fellowship, (3) quality assurance (QA) process, and (4) current US credentialing process.

Results: There was a $50 \%$ response rate. Fifty percent of the total respondents $(n=40)$ had an EUS fellowship program. Of the sites with an EUS fellowship, 36 had EUS fellowship-trained attendings. Of the sites without an EUS fellowship, 19 had EUS fellowship-trained faculty, $p \leq 0.0001$. Sites with an EUS fellowship had a greater percentage of staff credentialed to perform EUS as compared to sites with no EUS fellowship, $p=0.0161$. All sites with an EUS fellowship had EUS-credentialed attendings. In sites with an EUS fellowship, 35 conducted a formal QA of ED performed EUS scans versus 22 at sites without an EUS fellowship, $p=0.003$.

Conclusions: The survey results support hiring emergency attendings that have completed postgraduate training in emergency ultrasonography to aid in credentialing staff. This also seems to be helpful in completing a timelier QA of all ED ultrasounds.

Keywords: Emergency ultrasonography, Education, Survey, Credentialing, Quality assurance

\section{Background}

The use of emergency ultrasonography (EUS) has gained much popularity, since it was first introduced in the 1980s and is now a mainstay of diagnostic decision-making in

\footnotetext{
*Correspondence: devdas2@gmail.com

1 Department of Emergency Medicine, Northwell Health-Staten Island University Hospital, 475 Seaview Ave, Staten Island, NY 10305, USA Full list of author information is available at the end of the article
}

the emergency department [1]. In 2011, the Accreditation Council for General Medical Education (ACGME) Residency Review Committee (RRC) adopted an outcomes-based approach to residency accreditation in the United States. As part of this endeavor, EUS was deemed as one of the essential components in Patient Care, which is one of six core competencies for emergency medicine residency training. Since adoption of this mandate, every emergency medicine (EM) residency program must teach 
and assess ultrasound competency as part of its education [2]. As a result of this directive, improvements in US technology and research into new potential applications for US, the use of EUS has increased in the emergency department (ED). This expanded use is now highlighting the substantial issue of individual hospitals in credentialing its emergency medicine attending physicians in EUS in the United States. This issue is also of importance as an increasing number of hospitals is now requesting reimbursement for emergency ultrasounds.

Numerous organizations within EM have proposed guidelines on how to train physicians in EUS applications. Foremost among these are the 2008 American College of Emergency Physicians (ACEP) guidelines that recommend 150 ultrasound examinations in six specific applications [3]. However, several studies have shown that though there is an increase in EP training in EUS, there remains a wide variation in the type and extent of teaching in individual academic training programs $[4,5]$. This variability in teaching and education presents a challenging situation for EDs to credential attending physicians in the use of EUS.

An extensive review of the current literature has shown that no standardized method of ED attending credentialing in EUS exists in the United States. Furthermore, credentialing is primarily an individual hospital function. This lack of a universal credentialing process in EUS may lead to a discrepancy in the delineation of privileges for a physician from hospital to hospital, i.e., a physician credentialed in performing ultrasound in one hospital may not be credentialed to do so in another hospital [6]. The objective of this study is to gain an understanding of how many emergency departments are currently credentialing its attending staff in EUS, what the internal structure and staffing are of these emergency departments, and how they are currently performing quality assurance of the ultrasounds performed.

\section{Methods Study design}

This was a cross-sectional, web-based survey that was sent to 160 ACGME-accredited EM residency programs from July 2013 to November 2013. This was the total number of ACGME-accredited EM residency programs in existence during this time frame. A cover letter explaining the research study was initially sent to all EM residency program directors who were identified through the SAEM Residency Directory website. If deemed appropriate, the residency directors were instructed to share the survey with the US credentialing authority in their emergency department to acquire more accurate answers. A reminder email was sent 1 month after the initial survey request and again 2 months later to hospitals that had not completed the survey. The survey consisted of 23 questions created by the study authors regarding: (1) number of emergency attendings on staff, (2) presence or absence of an EUS fellowship, (3) quality assurance (QA) process, and (4) current EUS credentialing process (Figs. 1, 2). The survey required about 5-10 min to complete and included multiple choice and free text answers.

The survey was initially tested on a focus group consisting of three EUS program directors at various academic institutions. Questions were adjusted as suggested by the focus group for wording clarification purposes. Each academic institution that was sent a survey was provided with a unique numerical identifier after responses were obtained. All answers to the survey were de-identified and confidential prior to statistical analysis. This survey study was approved by the Institutional Review Board and was granted a waiver of signed consent at the authors' home institution.

\section{Data analysis}

The survey was distributed and results directly extracted from the online survey tool Surveymonkey@. Categorical data were analyzed utilizing descriptive statistics. The differences between groups were analyzed with the Chisquare analysis. Multivariate analysis was performed with a logistical regression model. Ordinal data were analyzed with a Mann-Whitney test and Spearman correlation.

\section{Results}

There was a $50 \%$ response rate to the survey study for a total of 80 respondents out of 160 programs (Table 1). All responses were from academic institutions in either a community $(n=22)$ or academic setting $(n=58)$. Of all respondents, $68.75 \%(n=55)$ had US-trained faculty on site. Fifty percent of total respondents $(n=40)$ had an EUS fellowship program. Of the sites with an EUS fellowship, 36 had EUS fellowship-trained attendings. Of the sites without an EUS fellowship, 19 had EUS fellowshiptrained faculty, $p \leq 0.0001$. Sites with an EUS fellowship had a greater percentage of staff credentialed to perform EUS as compared to sites with no EUS fellowship, $p=0.0161$.

\section{Ultrasound credentialing}

Ultrasound credentialing was done by a variety of methods at different sites. All sites with an EUS fellowship had EUS-credentialed attendings. The designated EUS director usually performed the process of credentialing at these sites. At sites without an EUS fellowship, 7 had no credentialing for emergency attendings to perform EUS in the emergency department, $p=0.025$ (Table 2). 
1. What position do you hold at your institution?
a. Ultrasound Director
b. Residency Director
c. Department Director
d. Other, please specify

2. How would you describe your institution?
a. Academic
b. Community
c. Rural
d. Urban
e. Suburban
f. Mixture

3. What is the annual volume of your Emergency Department?
a. $<50,000$ patient visits per year
b. 50,000-90,0000 patient visits per year
c. $>90,0000$ patient visits per year

4. What is the format of your residency program?
a. PGY I-III
b. PGY I-IV
c. PGY II-IV

5. Do you have any ultrasound-fellowship trained emergency physicians on staff?
a. Yes
b. No

6. How many emergency medicine attendings are on staff?

7. Are all of your emergency medicine attendings board-certified in emergency medicine?
a. Yes
b. No

8. If not, how many are not board-certified in emergency medicine?

9. Do you have a portable ultrasound machine in your Emergency Department?
a. Yes
b. No

10. If so, how often is the ultrasound machine available for use by your emergency medicine attending staff in your department?
a. All of the time
b. Some of the time
c. Very rarely

11. Roughly how many ultrasounds do your emergency medicine attending staff order through your radiology department yearly?
a. $<100$
b. $100-500$
c. $>500$

12. What type of ultrasounds does your attending staff mostly order through the Radiology Department? (Circle all that apply)
a. DVT
b. AAA
c. FAST
d. Torsion (ovarian and/or testicular)
e. Transabdominal/transvaginal pregnancy
f. Gallbladder
g. Cardiac
h. Renal
i. Musculoskeletal (including abscess, cellulitis or foreign body)
j. Lung
k. Ocular

Fig. 1 Survey questionnaire 
13. How often is the Radiology Department available to perform the ultrasounds ordered by your attending staff?
a. $<12$ hours per day
b. 12-24 hours
c. 24 hours a day

14. On average, how many TOTAL ultrasounds does your emergency medicine attending staff perform yearly on their own?
a. $<50$
b. $50-100$
c. $>100$

15. What percentage of your emergency medicine staff is credentialed to perform bedside ultrasound?
a. $0-25 \%$
b. $25-50 \%$
c. $50-75 \%$
d. $75-100 \%$

16. Who credentials your attending staff to perform emergency ultrasound?
a. Department Director
b. Ultrasound Director
c. Hospital credentialing board
d. We don't credential our staff in ultrasound
e. Other, please specify

17. If you are credentialing your staff to perform emergency ultrasound, what are some of the barriers you have come across in doing so?

18. What procedure do you use to credential your staff to perform emergency ultrasounds?

19. Is there a formal quality assurance (QA) process for ultrasounds performed by your emergency medicine attending staff?
a. Yes
b. No

20. If so, what kind of QA process do you use?
a. US scans reviewed weekly
b. US scans reviewed monthly
c. US scans reviewed yearly
d. US scans are not reviewed
e. Other, please specify

21. If a QA process exists, who performs the QA?
a. US director
b. Radiology department
c. Other, please specify

22. Do you currently submit ultrasounds performed by your emergency medicine attending staff for reimbursement?
a. Yes
b. No

23. If not, are there plans to do so in the future?
a. Yes
b. No

Fig. 2 Survey questionnaire

\section{Quality assurance}

In sites with an EUS fellowship, 35 conducted a formal QA of ED performed EUS scans versus 22 at sites without an EUS fellowship, $p=0.003$. At hospital sites with an EUS fellowship that conducted formal QA, $100 \%$ (35) had the QA process performed by the EUS director. In the non-EUS fellowship sites, 18 had the QA performed by the EUS director, $p=0.019$. At non-EUS fellowship sites without an EUS director who are conducting QA, 4 utilized the following for QA: (1) assigned faculty member, (2) program director/director of pediatric ED, (3) hospital EUS credentialing committee, or (4) radiology department. At hospitals with an EUS fellowship that conducted a formal QA, 29 conducted QA weekly or less, 5 conducted QA bi-weekly-to-monthly, and 1 conducted QA in another format. At non-EUS fellowship 
Table 1 Demographics of study population

\begin{tabular}{|c|c|}
\hline Respondents & $\mathrm{N}$ (percentage) \\
\hline \multicolumn{2}{|l|}{ Respondent demographics } \\
\hline Number of respondents & 80 \\
\hline \multicolumn{2}{|l|}{ Position in the institution } \\
\hline Ultrasound director & $23(28.75)$ \\
\hline Residency director & $42(52.5)$ \\
\hline Department director & $1(1.25)$ \\
\hline Other faculty & $16(20)$ \\
\hline \multicolumn{2}{|l|}{ Institution description } \\
\hline Academic & $58(72.5)$ \\
\hline Community & $22(27.5)$ \\
\hline \multicolumn{2}{|c|}{ Annual departmental volume (k visits) } \\
\hline$<50$ & $6(7.5)$ \\
\hline $50-90$ & $35(43.75)$ \\
\hline$>90$ & $40(50)$ \\
\hline \multicolumn{2}{|l|}{ Residency program format } \\
\hline PGY 1-3 & $53(66.25)$ \\
\hline PGY 1-4 & $26(32.5)$ \\
\hline EM/IM or EM/FM & $1(1.25)$ \\
\hline \multicolumn{2}{|l|}{ Ultrasound demographics } \\
\hline \multicolumn{2}{|c|}{ Does the site have portable ultrasound in the ED? } \\
\hline Yes & $80(100)$ \\
\hline No & $0(0)$ \\
\hline \multicolumn{2}{|c|}{ Does the site have an ultrasound fellowship? } \\
\hline Yes & $40(50)$ \\
\hline No & $40(50)$ \\
\hline \multicolumn{2}{|c|}{ Does the site have ultrasound trained faculty? } \\
\hline Yes & $55(68.75)$ \\
\hline No & $25(31.25)$ \\
\hline
\end{tabular}

sites, 5 conducted QA weekly or less, 13 conducted QA bi-weekly-to-monthly, 4 performed QA in another format (including rolling, yearly, or quarterly), $p<0.001$. (Table 2).

\section{Discussion}

The response rate to this survey study was on par with other studies conducted on similar topics. What differentiates this data set from other studies was that $50 \%$ of total respondents were affiliated with sites supporting EUS fellowship programs, while $50 \%$ were not. This allowed for a greater understanding of the difference in the credentialing and QA process at hospital sites with and without an EUS fellowship.

The survey results support that there is a great deal of variation in the number of EUS-credentialed staff and method for credentialing emergency attendings in EUS. This variability seemed to depend largely on the presence or absence of an EUS fellowship program at the
Table 2 Credentialing and quality assurance responses

\begin{tabular}{ll}
\hline Respondents & N (percentage) \\
\hline $\begin{array}{l}\text { Percentage of ED attendings credentialed for EUS } \\
0-25 \%\end{array}$ & $15(18.75)$ \\
$25-50 \%$ & $22(27.5)$ \\
$50-75 \%$ & $12(15)$ \\
$75-100 \%$ & $31(38.75)$ \\
Who credentials the staff to perform EUS? & $16(20)$ \\
Department director & $50(62.5)$ \\
Ultrasound director & $32(40)$ \\
Hospital credentialing board & $7(8.75)$ \\
Do not credential & $1(1.25)$ \\
Other & \\
Is there a formal QA process for EP performed US? & \\
Yes & $57(71.25)$ \\
No & $23(28.75)$ \\
If a QA process exists, who performs the QA? & \\
Ultrasound director & $56(98.25)$ \\
Radiology department & $1(1.75)$ \\
N/A & 23 \\
\hline
\end{tabular}

institution. Having an EUS fellowship and a dedicated EUS director seems to be associated with wider credentialing of attending staff. This is likely due to having a greater number of faculty members to provide the additional training required to credential staff. However, this does not necessarily apply to institutions that have EUS fellowship-trained faculty, but no EUS fellowship on site. In addition, institutions without an EUS fellowship and without an EUS director are largely not credentialing their faculty to perform ultrasounds.

Similarly, there is variability in the QA process at hospital sites with and without an EUS fellowship program. Based on the results of the survey, hospital sites with an EUS fellowship are more likely to perform a formal QA process. Overwhelmingly, the majority of the hospitals manage their QA process within the ED. However, at hospital sites without an EUS fellowship program, there was greater variability in the method by which the QA process is currently being performed. Of hospital sites with a formal QA process, ultrasounds are likely to be reviewed on a weekly basis. However, if no EUS fellowship program is present, there is a greater likelihood that the QA process is not being conducted on a weekly basis. However, studies have shown that a weekly QA process is the optimal method to provide feedback, performance improvement, and assurance that ultrasounds are being performed and interpreted correctly [7].

In contrast to other medical specialties that utilize ultrasound, the examinations performed by emergency physicians are highly focused, limited, and goal-directed 
[8]. As Jang, et al. put forth in his recent article regarding competency-based mandate in EUS, to be considered competent in EUS, physicians must have (1) an understanding of the disease process and indications for the diagnostic modality, (2) technical skills to acquire appropriate and interpretable images, (3) the ability to reliably interpret sonograms, and (4) management skills to apply the findings in light of their patient's clinical presentation [8]. The American Board of Emergency Medicine (ABEM) has been seeking to gain approval amongst its members to offer physician certification in clinical ultrasonography through the American Board of Medical Specialties (ABMS). ABEM envisions that once this is approved, physicians who do not achieve certification in clinical ultrasonography might eventually have a more difficult time practicing or billing for ultrasonography in some institutions. However, the process by which this may occur will require rigorous review through ABMS and may not occur for several years. As this move by ABEM highlights, it is becoming increasingly apparent that EUS is a vital skill in the ED management of patients. Currently, there exist evidence-based recommendations and expert consensus recommendations for specific applications for EUS, including for point-of-care lung and cardiac ultrasound $[9,10]$. There have also been well-researched and comprehensive proposed guidelines/curriculum for teaching EUS and providing a foundational knowledge of point-of-care ultrasonography. However, these guidelines have not been prospectively validated [11]. Therefore, training and assessment of ultrasound education among different residency programs vary greatly [12]. What this study poses is the understanding that having an EUS fellowship, or at least staff that have completed an EUS fellowship, is associated with increased training of attendings in emergency ultrasonography.

Another key factor that was highlighted in this study was the variability in which hospitals are currently performing their QA process. As hospitals are moving forward with more widespread use of EUS, many hospitals are already, or are soon planning, to request reimbursement for ultrasounds performed in the ED. Each US that is performed, used to make clinical decisions and are subsequently submitted for reimbursement require appropriate documentation regarding the indication for the examinations, written interpretation of the examinations, description of the organs or structures studied, who performed the examinations, and documentation of the scope of the study [13]. For patient safety purposes, it is, therefore, imperative that all of these studies be reviewed for QA on a timely basis. Having an EUS fellowship is likely conducive to having additional staff to review all of these ultrasounds and to ensure that the QA process is more streamlined.

\section{Limitations}

There are inherent limitations to any study based on survey results. This survey was subject to selection bias in that hospital sites that regularly utilize EUS are more likely to respond to the questionnaire. However, our response rate allowed for a unique data subset to analyze given that exactly $50 \%$ had an EUS fellowship program versus $50 \%$ that did not.

This study was also limited because hospital sites were chosen exclusively for having an ACGME-accredited EM residency program. This was done so that a greater understanding of the credentialing and QA process could be made at sites that are likely to be invested in having a robust EUS education program. However, results may be quite different if applied to hospital sites without an EM residency program and may not be generalizable to nonacademic institutions.

\section{Conclusions}

The spectrum of growth of EUS in daily practice within EDs nationwide is evident over the past several decades. This was exemplified by the RRC, which has made US education one subcomponent of its six emergency medicine core competencies. It is clear, therefore, that all hospitals, especially those with an ACGME-accredited residency program, will need to ensure that its staff is well versed in their US knowledge and technical skills. Credentialing their emergency attendings in EUS is a hospital-specific issue, but is a complex one when considering the extensive requirements that all physicians must meet to be considered competent in emergency ultrasonography. Our survey results seem to support hiring staff that has completed extra training in emergency ultrasonography to aid in credentialing other staff in EUS. This also seems to be helpful in completing a more timely QA process of all ultrasounds performed in the ED. In the future, we hope to look into what methods hospitals are currently using to credential their staff in EUS and which of these methods appear to be most effective.

\section{Abbreviations}

EUS: emergency ultrasonography; ACGME: Accreditation Council for General Medical Education; RRC: Residency Review Committee; EM: emergency medicine; ED: emergency department; ACEP: American College of Emergency Physicians.

\section{Authors' contributions}

DD contributed to the development of the concept of the research, research design, data acquisition, and development of manuscript. MK contributed to the development of the concept of the research and research design. $\mathrm{CB}$ contributed to the development of the concept of the research and research design. AN contributed to the development of the concept of the research, research design, and data acquisition. SG contributed to the development of the concept of the research, research design, statistical analysis, and development of manuscript. All authors read and approved the final manuscript. 


\section{Authors' information}

DD is the Associate Director of Emergency Ultrasound and Co-Fellowship Director in the Department of Emergency Medicine at Northwell HealthStaten Island University Hospital. She is currently involved in the development of the quality assurance process and credentialing of attending physicians in emergency ultrasound in her department.

MK is the Director of Emergency Ultrasound and Co-Fellowship Director in the Department of Emergency Medicine at Northwell Health-Staten Island University Hospital. She is currently involved in the development of the quality assurance process and credentialing of attending physicians in emergency ultrasound in her department.

$\mathrm{CB}$ is a member of the Emergency Ultrasound Division in the Department of Emergency Medicine at Northwell Health-Staten Island University Hospital. She is currently involved in the development of the quality assurance process and credentialing of attending physicians in emergency ultrasound in her department.

AN is an attending in the Department of Emergency Medicine at Northwell Health-Staten Island University Hospital.

SG is the Director of the Emergency Department at Northwell HealthFranklin Hospital.

\section{Author details}

1 Department of Emergency Medicine, Northwell Health-Staten Island University Hospital, 475 Seaview Ave, Staten Island, NY 10305, USA. ${ }^{2}$ Department of Emergency Medicine, Northwell Health-Franklin Hospital, 900 Franklin Ave, Valley Stream, NY 11580, USA.

\section{Competing interests}

The authors declare that they have no competing interests.

Received: 2 December 2015 Accepted: 11 May 2016

Published online: 26 May 2016

\section{References}

1. Moore CL, Molina AA, Lin H (2006) Ultrasonography in Community Emergency Departments in the United States; access to ultrasonography performed by consultants and status of emergency physician-performed ultrasonography. Ann Emerg Med 47(2):147-153
2. Jang TB, Coates WC, Liu YT (2012) The competency-based mandate for emergency bedside sonography training and a tale of two residency programs. J Ultrasound Med 31:515-521

3. American College of Emergency Physicians (ACEP) (2008) "Policy statement: emergency ultrasound guidelines."

4. Moore CL, Gregg S, Lambert M (2004) Performance, training, quality assurance, and reimbursement of emergency physician-performed ultrasonography at academic medical centers. J Ultrasound Med 23:459-466

5. Ahern M, Mallin MP, Weitzel S et al (2010) Variability in ultrasound education among emergency medicine residencies. West J Emerg Med 11(4):314-318

6. Lanoix R (1997) Credentialing issues in emergency ultrasonography. Emerg Med Clinics of North America 15(4):913-920

7. Budhram G, Elia T, Rathlev N (2013) Implementation of a successful incentive-based ultrasound credentialing program for emergency physicians. W J Emer Med 14(6):602-608

8. Jang TB, Coates WC, Liu YT (2012) The competency-based mandate for emergency bedside sonography training and a tale of two residency programs. J Ultrasound Med 31:515-521

9. Via G, Hussain A, Wells M et al (2014) International evidence-based recommendations for focused cardiac ultrasound. J Am Soc Echocardiogr 27(7):683

10. Volpicelli G, Elbarbary M, Blaivas M et al (2012) International evidencebased recommendations for point-of-care lung ultrasound. Intensive Care Med 38(4):577-591

11. Atkinson P, Bowra J, Lambert $\mathrm{M}$ et al (2015) International federation for emergency medicine point of care ultrasound curriculum. CJEM 17(2):161-170

12. Cohen JS, Teach SJ, Chapman J (2012) Bedside ultrasound education in pediatric emergency medicine fellowship programs in the United States. Pediatr Emerg Care 28(9):845-850

13. Cardenas E (2004) Emergency medicine ultrasound policies and reimbursement guidelines. Emerg Med Clinics of N Am 22(3):829-838

\section{Submit your manuscript to a SpringerOpen ${ }^{\circ}$ journal and benefit from:}

- Convenient online submission

- Rigorous peer review

- Immediate publication on acceptance

- Open access: articles freely available online

- High visibility within the field

- Retaining the copyright to your article 